\title{
Desenlaces perinatales en mujeres según IMC: estudio multicéntrico de hospitales públicos y privados
}

\section{Perinatal outcomes in women according to BMI: A multicentric study in private and public hospitals}

\author{
Aurea Z. Ramos-Hernández¹, Liliana Martínez-Nuñez², Ana León-López³ , Adriana de Hoyos-Garza ${ }^{4}$ y \\ Abraham Zavala-García ${ }^{1 *}$ \\ ${ }^{1}$ Ginecología y Obstetricia, Hospital Ángeles Pedregal, Ciudad de México; ${ }^{2}$ Departamento de Enfermedades Crónicas, Centro de Investigación en \\ Nutrición y Salud, Instituto Nacional de Salud Pública, Cuernavaca, Morelos; ${ }^{3}$ Ginecología y Obtetricia, Hospital General de México "Dr. Eduardo \\ Liceaga", Ciudad de México; ${ }^{4}$ Pediatría, Hospital Ángeles Pedregal, Ciudad de México. México
}

\begin{abstract}
Resumen
Introducción: El objetivo principal fue comparar desenlaces perinatales según el índice de masa corporal (IMC) de pacientes embarazadas y observar si este variaba entre la primera y segunda gesta. Metodología: Estudio retrospectivo, descriptivo y comparativo, transversal, de pacientes embarazadas, ingresadas para resolución del embarazo entre julio 2018 y marzo 2019. Se estudiaron dos centros, uno público y otro privado. Resultados: De las pacientes estudiadas, 53.55\% pertenecían a hospital privado y $46.44 \%$ a público. El peso neonatal fue mayor en relación a mayor peso materno, tanto en el hospital privado como en el público. El IMC de pacientes en su segunda gesta en comparación a la primera, fue similar en el hospital privado, mientras que aumentó en el hospital público. Conclusiones: El sobrepeso y la obesidad en mujeres embarazadas se asocian a mayor peso neonatal; sin embargo, el mayor IMC entre gestas subsecuentes también tiene un impacto que se debe estudiar a fondo para identificar los determinantes sociales y generar estrategias que eliminen este aumento.
\end{abstract}

Palabras clave: Resultado de embarazo. Neonato. Obesidad. Sobrepeso.

\section{Abstract}

Background: The primary aim of this study was to compare perinatal outcomes based on pregnant body mass index (BMI) and interpregnancy BMI. Materials and Methods: We performed a retrospective, descriptive, comparative, and cross-sectional study of 534 pregnant women, void of comorbidities, checked-in to the obstetrical ward from july 2018 to march 2019. This study was performed at a private and a public hospital. Results: Of the total study population, 53.55\% belonged to a private hospital and $46.44 \%$ to a public hospital. The main outcome measure was neonatal birthweight according to BMI category: We found a proportional relationship between maternal weight and neonatal birthweight in both public and private settings. Interpregnancy BMI was similar in the private hospital, but in the public hospital, maternal weight during second pregnancy was greater. Conclusions: Obesity and overweight during pregnancy is associated to a greater birthweight, however, an interpregnancy increase in BMI is an issue that still has requires further research to determine social strategies to diminish this issue in pregnancy.

Key words: Pregnancy outcomes. Neonate. Obesity. Overweight.

Correspondencia:

*Abraham Zavala-Garcia

E-mail: Abraham.zavala@europe.com
Disponible en internet: 22-06-2021 Rev Hosp Jua Mex. 2021;88(2):56-62

www.revistahospitaljuarez.com 1405-9622/○ 2021 Sociedad Médico-Quirúrgica del Hospital Juárez de México, A.C. Publicado por Permanyer. Este es un artículo open access bajo la licencia CC BY-NC-ND (http://creativecommons.org/licenses/by-nc-nd/4.0/). 


\section{Introducción}

La obesidad es un problema tan importante para la salud pública que ha desplazado a problemas tradicionales como la desnutrición y enfermedades infecciosas ${ }^{1}$. En México se reportó una prevalencia de obesidad en el $37.5 \%$ de mujeres en edad reproductiva ${ }^{2,3}$. En el mundo la prevalencia de sobrepeso y obesidad ha aumentado en todos los grupos etarios en las últimas décadas $^{4}$. La Organización Mundial de la Salud (OMS) define obesidad como el índice de masa corporal (IMC, peso $[\mathrm{kg}] /$ estatura $\left.[\mathrm{cm}]^{2}\right) \geq 30 \mathrm{~kg} / \mathrm{m}^{2}$, y el sobrepeso como un IMC de 25.0 a $29.9 \mathrm{~kg} / \mathrm{m}^{21,5}$. Se recomienda que la ganancia ponderal en el embarazo sea en relación al IMC previo al embarazo (pre-IMC), de tal forma: una gestante con IMC normal debería aumentar 11.5$16.0 \mathrm{~kg}$, con sobrepeso de 7.0 a $11.5 \mathrm{~kg}$ y con obesidad únicamente $6 \mathrm{~kg}^{1}$. El aumento desmesurado de peso aumenta el riesgo de peso neonatal anormal ${ }^{6-10}$. El aumento de peso de la mujer durante el embarazo y el elevado pre-IMC se relacionan con diabetes gestacional $(\mathrm{DMG})^{11}$, enfermedad hipertensiva, complicaciones tromboembólicas, cáncer ${ }^{4,12}$ y eventos obstétricos adversos (aborto espontáneo, infección, hemorragias, riesgos de anestesia) ${ }^{13}$; sin embargo, las complicaciones trascienden el embarazo y pudiesen generar problemas durante la lactancia, el puerperio y a lo largo de la vida del recién nacido $(R N)^{12,14,15}$; además, factores intrauterinos contribuyen al crecimiento fetal excesivo, por ejemplo, tanto madres con DMG como madres obesas, pero con tolerancia normal a la glucosa, tienen aumento de riesgo de tener $\mathrm{RN}$ grandes para su edad gestacional $(G E G)^{16}$. Las implicaciones sobre el peso del RN pueden resultar en parto pretérmino, productos con peso bajo al nacer (PBN), pequeño para edad gestacional $(P E G)^{17}$ y GEG. Aquellos con PBN se asocian a patologías endocrinas, cardiovasculares, respiratorias, urinarias 0 del sistema nervioso central ${ }^{4,6,17-20}$, mientras que aquellos con PEG tienen mayor incidencia de síndrome metabólico, enfermedad coronaria, infartos, densidad mineral ósea disminuida y osteoporosis ${ }^{6,19,21}$. Factores que determinan el aumento de peso durante la gestación son: pre-IMC, talla, etnicidad, edad, paridad, tabaquismo, estado socioeconómico, ingesta calórica diaria e incluso el sexo del producto ${ }^{12,16}$. Por otra parte, el aumento de peso entre el primero y segundo embarazo se ha relacionado con un aumento de complicaciones obstétricas, como preeclampsia (odds ratio [OR]: 2.09; intervalo de confianza [ICl] 95\%: 1.68-2.61) y DMG (OR: 1.78; IC 95\%: 1.52-2.08) ${ }^{11,15,22,23}$.
Un pre-IMC elevado y mayor ganancia ponderal durante el embarazo se relaciona con RN de mayor peso, asociados a riesgo de GEG y macrosomía 6,22 . Mujeres embarazadas con pre-IMC menor y menor ganancia ponderal se relaciona con $\mathrm{RN}$ de menor peso y $\mathrm{PEG}^{6,12}$.

El objetivo de este estudio fue determinar el peso neonatal con relación con el IMC de cada paciente, en un hospital privado y uno público. Además, se determinó la prevalencia de peso normal, sobrepesoy obesidad enestas poblaciones. Por último, se evaluó la diferencia en IMC y desenlace neonatal de primigestas y secundigestas.

\section{Material y métodos}

Se realizó un estudio retrospectivo, descriptivo, observacional y comparativo de tipo transversal. Se estudiaron 534 pacientes embarazadas sin comorbilidades, que ingresaron al servicio de tococirugía para resolución del embarazo por trabajo de parto o cesárea entre julio de 2018 y marzo de 2019. Este estudio multicéntrico se llevó a cabo en el Hospital General de Cuautla, Morelos (un centro afiliado a una universidad, institución pública y de referencia) y el Hospital Ángeles Pedregal en Ciudad de México (un centro afiliado a una universidad, institución privada). Se realizó una búsqueda de la lista nominal de las mujeres que acudieron al servicio de tococirugía durante el periodo y en los centros mencionados, localizando expedientes clínicos mediante el archivo hospitalario y así se recolectaron los datos de variables. Se incluyeron: a) aquellas mujeres embarazadas de término para resolución por parto o cesárea, b) con criterios de ingreso al área de tococirugía de uno de los dos centros evaluados, c) con historia clínica, exploración física y somatometría completa, d) con RN vivo al momento de la resolución del embarazo y d) que firmaron el consentimiento informado. Se excluyeron todas aquellas mujeres no embarazadas, aquellas con patologías sistémicas previas 0 durante el embarazo, con falta de firma de consentimiento informado o con antecedente de toxicomanías.

Para el análisis estadístico se utilizaron medidas de tendencia central, porcentajes (\%) y desviación estándar $(\sigma)$. Para el análisis inferencial se utilizó la prueba de normalidad Kolmogorov-Smirnov. La comparación de variables cuantitativas paramétricas se llevó a cabo por medio de la prueba $t$ de Student a dos colas para la comparación de matrices, analizado por el programa Stata versión 15.1 (Stata Corp., College Station, TX, EE.UU.). Todas las pacientes firmaron un consentimiento informado, corroborado por algún familiar y 
autorizado por un médico tratante. Al no contar con ningún tratamiento experimental, por el carácter retrospectivo y por el manejo confidencial de los datos del expediente clínico, no fue necesario conseguir autorización por parte del comité de bioética.

\section{Resultados}

Se incluyeron en el estudio 534 pacientes, el 53.6\% $(n=286)$ de ellas tratadas en una institución privada (Tabla 1). La media de edad fue mayor en el hospital privado, de manera no estadísticamente significativa.

\section{Índice de masa corporal en las embarazadas}

El IMC promedio, así como el de las categorías de peso normal, sobrepeso y obesidad fue similar entre ambos centros. En el hospital privado, 28.3\% tuvieron un IMC normal, $47.9 \%$ sobrepeso y $23.4 \%$ de obesidad, mientras que, en el hospital público, el $24.6 \%$ presentaban IMC normal, el $48.4 \%$ sobrepeso y el $27 \%$ obesidad.

Para el análisis de IMC por centro, los resultados del hospital privado fueron los siguientes (Tabla 2): Las pacientes del grupo de obesidad tenían una edad mayor en promedio que aquellas con IMC normal (edad promedio en el grupo de obesidad de 34.58 años vs. 32.32 años en pacientes de IMC normal, $\mathrm{p}=0.003$ ).

\section{Desenlaces obstétricos}

El número de gestaciones y la edad gestacional de resolución fueron similares en los tres grupos de IMC. El número de gestaciones fue mayor en el hospital público (2.21 vs. 1.73; $p=0.003$ ). En el hospital privado, las pacientes obesas se asociaban de manera estadísticamente significativa a productos con mayor peso neonatal ( 2.952 vs. $2,697 \mathrm{~g} ; \mathrm{p}=0.021$ ), en comparación con las pacientes de peso normal. El mismo análisis se llevó a cabo en las pacientes del hospital público (Tabla 3), encontrando una edad mayor en aquellas pacientes con sobrepeso, al igual que una mayor edad gestacional de interrupción. En comparación a las pacientes de peso normal, se encontró un peso mayor en los neonatos de las pacientes con sobrepeso, sin embargo, este grupo también tuvo una interrupción del embarazo a una edad gestacional mayor, de forma estadísticamente significativa. Se procedió a analizar las diferencias según los IMC entre ambas instituciones (Tabla 4). En el hospital público, el peso neonatal fue
Tabla 1. Características demográficas de la población en ambos centros ( $N=534)$

\begin{tabular}{|l|c|c|c|}
\hline Características & $\begin{array}{c}\text { Hospital } \\
\text { Ángeles } \\
\text { Pedregal } \\
\text { (privado) }\end{array}$ & $\begin{array}{c}\text { Hospital } \\
\text { General de } \\
\text { Cuautla } \\
\text { (público) }\end{array}$ & $\begin{array}{c}\text { Comparativo } \\
\text { Priv. vs. Púb. }\end{array}$ \\
\cline { 2 - 4 } & Valores & Valores* & Valor p $^{\dagger}$ \\
\hline $\mathrm{n}(\%)$ & $286(53.55)$ & $248(46.44)$ & - \\
\hline Edad, años & $33.32 \pm 4.52$ & $28.35 \pm 5.74$ & 1.743 \\
\hline $\begin{array}{l}\text { IMC, kg/m² } \\
\text { Peso normal } \\
\text { Sobrepeso } \\
\text { Obesidad }\end{array}$ & $27.65 \pm 4.00$ & $28.15 \pm 4.35$ & 0.169 \\
\hline $\begin{array}{l}\text { Gestaciones, } \\
\text { número }\end{array}$ & $1.73 .41 \pm 1.23$ & $23.19 \pm 1.29$ & 0.416 \\
\hline
\end{tabular}

*Media \pm desviación estándar.

'Valores de promedios analizados por t de Student a dos colas.

${ }^{\ddagger}$ Valor estadísticamente significativo (valor $p<0.05$ ).

IMC: índice de masa corporal.

mayor en los tres grupos, peso normal, sobrepeso y obesidad (2,925 vs. $2,697 \mathrm{~g}, \mathrm{p}=0.013 ; 3,070$ vs. 2,815 $g, p=0.0001$; y 3,277 vs. $2,953 \mathrm{~g}, \mathrm{p}=0.0009$, respectivamente). Esto se asoció a un IMC ligeramente mayor (no significativo) y una edad de resolución mayor. Por otra parte, el valor de APGAR al primer minuto fue mayor en los RN del hospital privado en todos los grupos de IMC, de manera estadísticamente significativa, en comparación con el hospital público.

\section{Índice de masa corporal en secundigestas}

Por último, se analizó la diferencia entre pacientes que acudieron a la resolución de su primer embarazo, en comparación con aquellas que cursaban su segunda gestación (Tabla 5), en ambos centros. Se encontró que la edad de estas pacientes era mayor de manera estadísticamente significativa en aquellas de hospital privado (32.36 vs. 34.4 años; $p=0.0005$ ) y similar en aquellas del medio público. Las pacientes de hospital privado presentaron un IMC prácticamente igual entre primigestas y secundigestas, mientras que en el hospital público, las secundigestas presentaban un IMC mayor estadísticamente significativo en comparaciónalas primigestas (28.81 vs. $26.83 \mathrm{~kg} / \mathrm{m}^{2} ; \mathrm{p}=0.004$ ).

\section{Discusión y conclusiones}

Un estudio realizado en adolescentes mexicanas reportó que el pre-IMC de un grupo de 601 mujeres 
Tabla 2. Características obstétricas y neonatales de la población de hospital privado ( $\mathrm{n}=286$ )

\begin{tabular}{|c|c|c|c|c|c|}
\hline \multicolumn{6}{|c|}{ Hospital privado, población total $(\mathrm{n}=\mathbf{2 8 6}$ ) } \\
\hline Variables & Peso normal ${ }^{*}$ & Sobrepeso* & Valor $\mathbf{p}^{\dagger}$ & Obesidad $^{+}$ & Valor $\mathbf{p}^{\dagger}$ \\
\hline$n(\%)$ & $81(28.32)$ & $137(47.90)$ & - & $67(23.42)$ & - \\
\hline Edad, años & $32.32 \pm 5.03$ & $33.31 \pm 4.16$ & 0.136 & $34.58 \pm 4.33$ & $0.003^{\ddagger}$ \\
\hline IMC, kg/m2 & $23.37 \pm 1.23$ & $27.41 \pm 1.32$ & 1.163 & $33.29 \pm 2.94$ & 2.795 \\
\hline Gestaciones, número & $1.75 \pm 1.23$ & $1.69 \pm 0.87$ & 0.589 & $1.85 \pm 0.91$ & 0.511 \\
\hline Edad gestacional, SDG & $37.86 \pm 2.79$ & $37.14 \pm 2.76$ & 0.491 & $37.19 \pm 2.53$ & 0.478 \\
\hline \multicolumn{6}{|c|}{ Resultados neonatales } \\
\hline Peso, gramos & $2,697.58 \pm 706.64$ & $2,815.29 \pm 645.08$ & 0.222 & $2,952.25 \pm 625.86$ & $0.021^{\ddagger}$ \\
\hline APGAR 1 (primer minuto) & $8.74 \pm 1.10$ & $8.69 \pm 1.03$ & 0.717 & $8.66 \pm 1.19$ & 0.659 \\
\hline APGAR 5 (quinto minuto) & $9.06 \pm 1.11$ & $8.96 \pm 0.95$ & 0.477 & $8.91 \pm 1.51$ & 0.497 \\
\hline
\end{tabular}

*Media \pm desviación estándar.

${ }^{\dagger}$ Valores de promedios analizados por $t$ de Student a dos colas.

‡Valor estadísticamente significativo $(\mathrm{p}<0.05)$.

IMC: índice de masa corporal; SDG: semanas de gestación.

Tabla 3. Características obstétricas y neonatales de la población de hospital público ( $\mathrm{n}=248$ )

\begin{tabular}{|c|c|c|c|c|c|}
\hline \multicolumn{6}{|c|}{ Hospital público, población total ( $\mathrm{n}=248$ ) } \\
\hline Variables & Peso normal ${ }^{*}$ & Sobrepeso* & Valor $\mathrm{p}^{\dagger}$ & Obesidad $^{+}$ & Valor $\mathbf{p}^{\dagger}$ \\
\hline $\mathrm{n}(\%)$ & $61(24.59)$ & $120(48.38)$ & & $67(27.01)$ & \\
\hline Edad, años & $21.15 \pm 5.36$ & $22.86 \pm 5.09$ & $0.041^{\ddagger}$ & $26.22 \pm 6.09$ & 1.758 \\
\hline IMC, $\mathrm{kg} / \mathrm{m}^{2}$ & $23.19 \pm 1.29$ & $27.53 \pm 1.40$ & 2.544 & $33.78 \pm 3.17$ & 1.086 \\
\hline Gestaciones, número & $2.07 \pm 4.02$ & $1.92 \pm 1.21$ & 0.778 & $2.87 \pm 1.68$ & 0.152 \\
\hline Edad gestacional, SDG & $38.78 \pm 1.57$ & $39.36 \pm 1.44$ & $0.018^{\ddagger}$ & $39.01 \pm 1.38$ & 0.379 \\
\hline \multicolumn{6}{|l|}{ Resultados neonatales } \\
\hline Peso, gramos & $2,925.23 \pm 364.49$ & $3,070.48 \pm 386.52$ & $0.014^{\ddagger}$ & $3,277.61 \pm 471.90$ & 2.775 \\
\hline APGAR 1 (primer minuto) & $8.30 \pm 0.72$ & $8.33 \pm 0.81$ & 0.746 & $8.27 \pm 0.77$ & 0.840 \\
\hline APGAR 5 (quinto minuto) & $8.98 \pm 0.13$ & $8.94 \pm 0.37$ & 0.269 & $8.94 \pm 0.30$ & 0.277 \\
\hline
\end{tabular}

*Media \pm desviación estándar.

tValores de promedios analizados por $t$ de Student a dos colas.

"Valor estadísticamente significativo $(\mathrm{p}<0.05)$.

IMC: índice de masa corporal; SDG: semanas de gestación.

adolescentes presentó un $11.5 \%$ de pacientes con sobrepeso y un $10.1 \%$ con obesidad. Sin embargo, una vez embarazadas, el $27.6 \%$ tuvo un aumento de peso excesivo durante la gestación ${ }^{24}$. Según nuestro estudio, la distribución de IMC normal, sobrepeso y obesidad fue muy similar en hospital privado y público.

Es importante dilucidar entre los tipos de atención (público y privado), pues pueden existir importantes diferencias en el contexto obstétrico. Por ejemplo, en un estudio de cohorte en Australia (Adams, et al., 2017) se reportó que había una mayor asociación a mortalidad perinatal y neonatal en un hospital público en comparación a un privado con una OR ajustada de 1.53, IC 95\%: $1.29-1.80$ y 1.48 , IC $95 \%$ : $1.15-1.89$, respectivamente ${ }^{25}$. Nuestro estudio muestra resultados diferentes de peso neonatal entre instituciones.

El número de gestaciones fue mayor en el hospital público que en el privado. Esto es relevante en el 
Tabla 4. Características obstétricas y neonatales de la población de hospitales privado y público, según IMC ( $n=534$ )

\begin{tabular}{|c|c|c|c|c|c|c|c|c|c|}
\hline \multirow[t]{2}{*}{ Cat. Según IMC } & \multicolumn{3}{|c|}{ Normal } & \multicolumn{3}{|c|}{ Sobrepeso } & \multicolumn{3}{|c|}{ Obesidad } \\
\hline & Privado* & Público* & $\begin{array}{c}\text { Valor } \\
\mathbf{p}^{\dagger}\end{array}$ & Privado* & Público* & $\begin{array}{c}\text { Valor } \\
\mathbf{p}^{\dagger}\end{array}$ & Privado* $^{*}$ & Público* & $\begin{array}{c}\text { Valor } \\
p^{\dagger}\end{array}$ \\
\hline Edad, años & $\begin{array}{c}32.32 \pm \\
5.03\end{array}$ & $\begin{array}{c}21.15 \pm \\
5.36\end{array}$ & 1.776 & $\begin{array}{c}33.31 \pm \\
4.16\end{array}$ & $\begin{array}{c}22.86 \pm \\
5.09\end{array}$ & 2.085 & $34.58 \pm 4.33$ & $26.22 \pm 6.09$ & 1.856 \\
\hline IMC, kg/m² & $\begin{array}{c}23.37 \pm \\
1.23\end{array}$ & $\begin{array}{c}23.19 \pm \\
1.29\end{array}$ & 0.415 & $\begin{array}{c}27.41 \pm \\
1.32\end{array}$ & $\begin{array}{c}27.53 \pm \\
1.40\end{array}$ & 0.495 & $33.29 \pm 2.94$ & $33.78 \pm 3.17$ & 0.350 \\
\hline $\begin{array}{l}\text { Gestaciones, } \\
\text { número }\end{array}$ & $\begin{array}{c}1.75 \pm \\
1.23\end{array}$ & $\begin{array}{c}2.07 \pm \\
4.02\end{array}$ & 0.552 & $\begin{array}{c}1.69 \pm \\
0.87\end{array}$ & $\begin{array}{c}1.92 \pm \\
1.21\end{array}$ & 0.084 & $1.85 \pm 0.91$ & $2.87 \pm 1.68$ & 1.598 \\
\hline $\begin{array}{l}\text { Edad } \\
\text { gestacional, SDG }\end{array}$ & $\begin{array}{c}37.86 \pm \\
2.79\end{array}$ & $\begin{array}{c}38.78 \pm \\
1.57\end{array}$ & 2.026 & $\begin{array}{c}37.14 \pm \\
2.76\end{array}$ & $\begin{array}{c}39.36 \pm \\
1.44\end{array}$ & 2.278 & $37.19 \pm 2.53$ & $39.01 \pm 1.38$ & 1.058 \\
\hline \multicolumn{10}{|c|}{ Resultados Neonatales } \\
\hline Peso, gramos & $\begin{array}{l}2,697.58 \\
\pm 706.64\end{array}$ & $\begin{array}{l}2,925.23 \\
\pm 364.49\end{array}$ & $0.013^{\ddagger}$ & $\begin{array}{l}2,815.29 \\
\pm 645.08\end{array}$ & $\begin{array}{l}3,070.48 \\
\pm 386.52\end{array}$ & $0.0001^{\ddagger}$ & $2,952.25 \pm 625.86$ & $3,277.61 \pm 471.90$ & $0.0009^{\ddagger}$ \\
\hline $\begin{array}{l}\text { APGAR } 1 \text { (primer } \\
\text { minuto) }\end{array}$ & $\begin{array}{c}8.74 \pm \\
1.10\end{array}$ & $\begin{array}{c}8.30 \pm \\
0.72\end{array}$ & $0.004^{\ddagger}$ & $\begin{array}{c}8.69 \pm \\
1.03\end{array}$ & $\begin{array}{c}8.33 \pm \\
0.81\end{array}$ & $0.002^{\ddagger}$ & $8.66 \pm 1.19$ & $8.27 \pm 0.77$ & $0.026^{\ddagger}$ \\
\hline $\begin{array}{l}\text { APGAR } 5 \text { (quinto } \\
\text { minuto) }\end{array}$ & $\begin{array}{c}9.06 \pm \\
1.11\end{array}$ & $\begin{array}{c}8.98 \pm \\
0.13\end{array}$ & 0.532 & $\begin{array}{c}8.96 \pm \\
0.95\end{array}$ & $\begin{array}{c}8.94 \pm \\
0.37\end{array}$ & 0.869 & $8.91 \pm 1.51$ & $8.94 \pm 0.30$ & 0.874 \\
\hline
\end{tabular}

Tabla 5. Características obstétricas y neonatales de la población de hospitales privado y público, según diferencias en mujeres primigestas y secundigestas

\begin{tabular}{|c|c|c|c|c|c|c|}
\hline \multirow[t]{2}{*}{ Variables } & \multicolumn{3}{|c|}{ Privado } & \multicolumn{3}{|c|}{ Público } \\
\hline & Primigestas $^{*}$ & Secundigestas ${ }^{*}$ & Valor-Pt & Primigestas $^{*}$ & Secundigestas* & Valor-P ${ }^{\dagger}$ \\
\hline $\mathrm{n}(\%)$ & 142 & 91 & - & 119 & 47 & - \\
\hline Edad & $32.36 \pm 4.11$ & $34.40 \pm 4.45$ & $0.0005^{\ddagger}$ & $20.18 \pm 4.66$ & $23.66 \pm 4.20$ & 1.077 \\
\hline IMC & $27.48 \pm 3.55$ & $27.58 \pm 4.16$ & 0.852 & $26.83 \pm 3.78$ & $28.81 \pm 3.98$ & $0.004^{\ddagger}$ \\
\hline Edad Gestacional & $37.5 \pm 2.03$ & $37.12 \pm 3.03$ & 0.297 & $39.23 \pm 1.41$ & $38.95 \pm 1.80$ & 0.337 \\
\hline Peso & $2,873.89 \pm 606.32$ & $2,855.12 \pm 687.70$ & 0.831 & $3,011.03 \pm 381.47$ & $3,068.91 \pm 410.66$ & 0.406 \\
\hline APGAR 1 (Primer minuto) & $8.80 \pm 0.60$ & $8.57 \pm 1.42$ & 0.155 & $8.29 \pm 0.75$ & $8.30 \pm 0.86$ & 0.979 \\
\hline APGAR 5 (Quinto minuto) & $9.12 \pm 0.50$ & $8.81 \pm 1.59$ & 0.077 & $0.8 .95 \pm 0.31$ & $8.94 \pm 0.32$ & 0.808 \\
\hline
\end{tabular}

hospital público, ya que las pacientes secundigestas tuvieron un IMC mayor que las primigestas. Esta diferencia de IMC entre gestaciones puede repercutir en eventos obstétricos adversos. Una revisión sistemática realizada por Martínez-Hortelano, et al. (2020) reportó que el aumento de peso entre una gestación y otra se asocia a un mayor riesgo de enfermedades hipertensivas inducidas por el embarazo (EHIE); por el contrario, la disminución de peso entre gestaciones se asocia a un menor riesgo de estas. Más aún, el 
aumento de solo $1 \mathrm{~kg} / \mathrm{m}^{2}$ se asocia a un riesgo $31 \%$ mayor de presentar EHIE en una gestación subsecuente $^{26}$. De la misma manera, en una revisión sistemática llevada a cabo por Teulings, et al. (2019) se reportó que el aumento entre 1-2 unidades de IMC entre gestaciones consecutivas se asociaba a un aumento del $51 \%$ para el desarrollo de DMG (ORa: 1.51; IC 95\%: 1.22-1.80) $)^{27}$.

En contraste con el aumento de peso intergestacional del hospital público, el hospital privado no tuvo este mismo aumento. Un estudio realizado por McBain, et al. (2016) reportó que en promedio las mujeres con un IMC normal ganaron $1 \mathrm{~kg} / \mathrm{m}^{2}$ entre el primer y segundo embarazo, mientras que las mujeres con sobrepeso $\mathrm{u}$ obesidad ganaron $1.37 \mathrm{~kg} / \mathrm{m}^{22}$.

Pacientes con IMC mayor se asociaron a pesos neonatales mayores. Esto se reflejó en el hospital privado en aquellas con obesidad, sin embargo, en el hospital público esta tendencia se observó en pacientes con sobrepeso. En un metaanálisis de Goldstein, et al. (2017), estos reportaron que la obesidad se asociaba a un mayor riesgo de GEG, un resultado similar a nuestro estudio y en congruencia con revisiones sistemáticas previas ${ }^{28}$. Más aún, reportaron que la obesidad clase I de la OMS tenía la mayor asociación la GEG (OR: 1.86; IC 95\%: 1.75-2.00)28. En el hospital público, los pesos neonatales promedio fueron mayores que en el hospital privado. Sin embargo, también la edad gestacional de interrupción fue mayor, por lo que es difícil atribuirlo exclusivamente al IMC materno.

Una limitación es el tamaño de muestra que se utilizó para este estudio, así como el diseño retrospectivo del estudio. Habría aportado información valiosa el contar con el IMC previo al embarazo para calcular el aumento ponderal durante el embarazo y al momento de la interrupción.

\section{Conclusiones}

En este estudio se demuestra la relación entre sobrepeso y obesidad con el peso neonatal, y las diferencias que existen en el IMC de las mujeres que cursan su primer segundo embarazo en el contexto privado y público, creando la oportunidad para investigar más este fenómeno y desarrollar estrategias de salud pública que permitan cambiar esta situación y eliminar los determinantes sociales relacionados con la problemática, disminuyendo las implicaciones obstétricas y neonatales que tienen el sobrepeso, la obesidad y el aumento de IMC entre gestaciones consecutivas, al mismo tiempo que se intenta disminuir la prevalencia de sobrepeso y obesidad en mujeres embarazadas, en edad reproductiva y en la población en general.

\section{Financiamiento}

La presente investigación no ha recibido ayudas específicas provenientes de agencias del sector público, sector comercial o entidades sin ánimo de lucro.

\section{Conflicto de intereses}

Los autores declaran no tener conflicto de intereses.

\section{Responsabilidades éticas}

Protección de personas y animales. Los autores declaran que para esta investigación no se han realizado experimentos en seres humanos ni en animales.

Confidencialidad de los datos. Los autores declaran que han seguido los protocolos de su centro de trabajo sobre la publicación de datos de pacientes.

\section{Derecho a la privacidad y consentimiento infor-} mado. Los autores han obtenido el consentimiento informado de los pacientes y/o sujetos referidos en el artículo.

\section{Bibliografía}

1. Catalano PM. Management of obesity in pregnancy. Obstet Gynecol. 2007;109:419-33.

2. Gutiérrez JP, Rivera-Dommarco J, Shamah-Levy T, Villalpando-Hernández S, Franco A, Cuevas-Nasu L, et al. Encuesta Nacional de Salud y Nutrición 2012. Resultados Nacionales. Cuernavaca, México: Instituto Nacional de Salud Pública; 2012.

3. Arredondo A, Torres C, Orozco E, Pacheco S, Aragón A, Huang F, et al. Indicadores socioeconómicos de la obesidad materna en México y Francia. Análisis comparado de dos cohortes. Rev Salud Publica. 2018; 20(2):245-53.

4. Wei Y-M, Yang H-X, Zhu W-W, Liu X-Y, Meng W-Y, Wang, Y-Q, et al. Risk of adverse pregnancy outcomes stratified for pre-pregnancy body mass index. J Matern Fetal Neonatal Med. 2016;29(13):2205-9.

5. DiBonaventura MD, Meincke H, Le Lay A, Fournier J, Bakker E, Ehrenreich $A$. Obesity in Mexico; prevalence, comorbidities, associations with patient outcomes, and treatment experiences. Diabetes Metab Syndr Obes. 2017;11:1-10

6. Du M-K, Ge L, Zhou M, Ying J, Qu F, Dong M-Y, et al. Effects of pre-pregnancy body mass index and gestational weight gain on neonatal birth weight. J Zhejiang Univ Sci B. 2017;18(3):263-71.

7. Liu Y, Dai W, Dai X, Li Z. Prepregnancy body mass index and gestational weight gain with the outcome of pregnancy: a 13-year study of 292 568 cases in China. Arch Gynecol Obstet. 2012;286(4):905-11.

8. Li N, Liu E, Guo J, Pan L, Li B, Wang P, et al. Maternal prepregnancy body mass index and gestational weight gain on pregnancy outcomes. PLoS One. 2013;8(12):e82310.

9. Sewell MF, Huston-Presley L, Super DM, Catalano P. Increased neonatal fat mass, not lean body mass, is associated with maternal obesity. Am J Obstet Gynecol. 2006;195:1100-3.

10. Hull HR, Dinger MK, Knehans AW, Thompson DM, Fields DA. Impact of maternal body mass index on neonate birthweight and body composition. Am J Obstet Gynecol. 2008;198(4):416.e1-416.e6. 
11. Nitert MD, Foxcroft KF, Lust K, Fagermo N, Lawlor DA, O'Callaghan M, et al. Overweight and obesity knowledge prior to pregnancy: a survey study. BMC Pregnancy Childbirth. 2011;11:96

12. Akgun N, Keskin $\mathrm{H}$, Ustuner I, Pekcan G, Avsar A. Factors affecting pregnancy weight gain and relationships with maternal/fetal outcomes in Turkey. Saudi Med J. 2017;38(5):503-8

13. Seligman LC, Duncan BB, Branchtein L, Gaio DSM, Mengue SS, Schmidt MI. Obesity and gestational weight gain: cesarean delivery and labor complications. Rev Saude Publica. 2006;40(3):457-65.

14. Ghaffari N, Srinivas SK, Durnwald CP. The multidisciplinary approach to the care of the obese parturient. Am J Obstet Gynecol. 2015;213(3):318-25.

15. Ding X-X, Xu S-J, Hao J-H, Huang K, Su P-Y, Tao F-B. Maternal pre-pregnancy $\mathrm{BMI}$ and adverse pregnancy outcomes among Chinese women: Results from the C-ABCS. Obstet Gynecol. 2016;36(3):328-32.

16. Barbour LA, Hernandez TL. Maternal non-glycemic contributors to fetal growth in obesity and gestational diabetes: spotlight on lipids. Curr Diab Rep. 2018;18:37.

17. Vesco KK, Sharma AJ, Dietz PM, Rizzo JH, Callaghan WM, England L, et al. Newborn size among obese women with weight gain outside the 2009 Institute of Medicine recommendation. Obstet Gynecol. 2011;117(4):812-8

18. Negrato CA, Gomes MB. Low birth weight: Causes and consequences. Diabetol Metab Syndr. 2013;5:49

19. Johnsson IW, Haglund B, Ahlsson F, Gustafsson J. A high birth weight is associated with increased risk of type 2 diabetes and obesity. Pediatr Obes. 2015;10(2):77-83.

20. Nodine PM, Hastings-Tolsma M. Maternal obesity: Improving pregnancy outcomes. MCN Am J Matern Child Nurs. 2012;37(2):110-5.
21. Scholtens DM, Bain JR, Reisetter AC, Muehlbauer MJ, Nodzenski M Stevens RD, et al. Metabolic networks and metabolites underlie associations between maternal glucose during pregnancy and newborn size at birth. Diabetes. 2016;65(7):2039-50.

22. McBain RD, Dekker GA, Clifton VL, Mol BW, Grzeskowiak LE. Impact of inter-pregnancy BMI change on perinatal outcomes: a retrospective cohort study. Eur J Obstet Gynecol Reprod Biol. 2016;205:98-104.

23. Mission JF, Marshall NE, Caughey AB. Pregnancy risks associated with obesity. Obstet Gyncol Clin North Am. 2015;42(2):335-53.

24. Sámano R, Chico-Barba G, Martínez-Rojano H, Godinez E, Rodriguez-Ventura AL, Avila-Koury G, et al. Prepregnancy body mass index classification and gestational weight gain on neonatal outcomes in adolescent mothers: A follow-up study. PLoS One. 2018; 13(7):e0200361.

25. Adams N, Tudehope D, Gibbons K, Flenady V. Perinatal mortality disparities betweem public care and private obstetrician-led care: a propensity score analysis. BJOG. 2018;125(2):149-58.

26. Martínez-Hortelano JA, Cavero-Redondo I, Álvarez-Bueno C, Sanabria-Martínez G, Poyatos-León R, Martínez-Vicaíno V. Interpregnancy weight change and hypertension during pregnancy, asystematic review and meta-analysis. Obstet Gynecol. 2020;135(1):68-79.

27. Teulings NEWD, Masconi KL, Ozanne SE, Aiken CE, Wood AM. Effect of interpregnancy weight change on perinatal outcomes: Systematic review and meta-analysis. BMC Pregnancy Childbirth. 2019;19:386.

28. Goldstein RF, Abell SK, Ranshina S, Misso M, Boyle JA, Black MH, et al. Association of gestational weight gain with maternal and infant outcomes. A systematic review and metaanalysis. JAMA. 2017; 317(21):2207-25. 